P. Jiménez Guerra and B. Rodríguez-Salinas

Nagoya Math. J.

Vol. 85 (1982), 81-86

\title{
STRICTLY LOCALIZABLE MEASURES
}

\author{
P. JIMÉNEZ GUERRA AND B. RODRÍGUEZ-SALINAS
}

\section{Introduction}

In this paper it is proved that every locally strictly localizable Radon measure of type $(\mathscr{H})$, is strictly localizable, from where it follows immediately the existence of lifting for these measures.

R. Ryan states in [9] that a complete measure has a lifting if and only if it is strictly localizable. The existence of lifting for the Lebesgue measure in $\boldsymbol{R}^{n}$ has been proved by von Neumann [4] and for general $\boldsymbol{\sigma}$ finite measures by D. Maharam [3]. A. and C. Ionescu Tulcea [2] have proved the existence of lifting for positive Radon measures in locally compact spaces, and L. Schwartz [10] has solved the problem for locally finite Radon measures (of type $(\mathscr{K})$ ) in arbitrary topological Hausdorff spaces.

B. Rodríguez-Salinas and P. Jiménez Guerra [7] and [8] have proved that every locally $\sigma$-finite Radon measure of type $(\mathscr{H})$ is strictly localizable, result which is an immediate consequence of the Maharam's theorem and of the theorem 2 in this paper (see Corollary 3).

Proposition 4 allows to extend, for locally strictly localizable Radon measures of type $(\mathscr{H})$, many results which are known for finite Radon measures of type $(\mathscr{H})$.

The results concerning the existence of different types of liftings for locally $\sigma$-finite Radon measures of type $(\mathscr{H})$, that were obtained by Rodríguez-Salinas in [6], can be easily extended for locally strictly localizable Radon measures of type ( $\mathscr{H})$, using Theorem 2 and Proposition 4 of this work.

\section{Notations and fundamentals}

We will denote by $\mathrm{E}$ an arbitrary topological space (Hausdorff or not) and by $\mathscr{H}$ a class of closed subsets of $E$. If $\mu$ is a Radon measure of

Received July 16, 1979. 
type $(\mathscr{H})$ on $E$ and $A \subset E$ we will denote by $\mu_{A}$ the Radon measure of type $\left(\mathscr{H}_{A}\right)$ on $A$, induced by the measure $\mu$ (see Theorem 78 of [7]).

By $\mu$-compact set and Radon measure of type $(\mathscr{H})$ we will understand the same as in [5].

A Radon measure $\mu$ of type $(\mathscr{H})$ on a topological space $E$ is strictly localizable (Definition 8, p. 16 and 17 of [2]) if and only if there exists a family $\mathscr{C}$ of $\mu$-measurable disjoint subsets of $E$, with positive and finite measure which verify one of the two following equivalent conditions:

$\mathrm{M}_{1}$. $\sup \{\tilde{K}: K \in \mathscr{C}\}=E$ (where $\tilde{K}$ is the equivalence class of the set $K$ with respect to the equivalence relation:

$$
A \equiv B \Leftrightarrow \mu(A \triangle B)=0,
$$

being $A$ and $B \mu$-measurable subsets of $E$ ).

$\mathrm{M}_{2}$. For every set $A \subset E$ with $\mu(A)<+\infty$, there is a countable subset $\mathscr{C}_{A} \subset \mathscr{C}$ such that $A-\cup_{K \in \mathscr{Q}_{A}} K$ is $\mu$-negligible.

From now on we will say that $\mathscr{C}$ is a family of strict localizability for $\mu$ and we will denote by $\overline{\mathscr{C}}$ the set $\bigcup_{K \in \mathscr{E}} K$.

LemMa 1. If $\mu$ is a locally strictly localizable Radon measure of type $(\mathscr{H})$ on $E, G$ is an open subset of $E$ such that $\mu(E-G)>0$ and $\mathscr{C}$ is a family of strict localizability for $\mu_{G}$, then there exists an open subset $G^{\prime}$ of $E$ and a family $\mathscr{C}^{\prime}$ of strict localizability for $\mu_{G^{\prime}}$, such that $\mathscr{C} \subset \mathscr{C}^{\prime}$ and $G$ is strictly contained in $G^{\prime}$.

Proof. We have that $\mu(E-G)>0$, then there exists a set $H \in \mathscr{H}$ of measure $\mu(H)>0$, such that $H \subset E-G$. Since $H$ is $\mu$-comact, $\mu$ is locally strictly localizable and $\mu(H)>0$, it is easily deduced the existence of an open subset $U$ of $E$ such that $\mu_{U}$ is strictly localizable and $\mu(U \cap H)>0$. Evidently, $G$ is strictly contained in $G^{\prime}=G \cup U$.

Let $\mathscr{D}$ be a family of strict localizability for $\mu_{U}$. For every subset $\mathscr{S}$ $\subset \mathscr{D}$ we set

$$
\mathscr{S}^{\prime}=\{K-G: K \in \mathscr{S}\}
$$

and

$$
\mathscr{S}^{\prime \prime}=\left\{K^{\prime} \in \mathscr{S}^{\prime}: \mu\left(K^{\prime}\right)>0\right\} .
$$

We will prove now that $\mathscr{C}^{*}=\mathscr{C} \cup \mathscr{D}^{\prime \prime}$ is a family of strict localizability for $\mu_{G^{\prime}}$ for which it is enough to verify that $\mathscr{C}^{*}$ satisfies $\mathrm{M}_{2}$.

If $A \subset G^{\prime}$ and $\mu_{G^{\prime}}(A)<+\infty$ then $\mu_{G}(A \cap G)$ and $\mu_{U}(A \cap U)$ are finite 
and there exist two countable subsets $\mathscr{C}_{A} \subset \mathscr{C}$ and $\mathscr{D}_{A} \subset \mathscr{D}$ such that

$$
\mu_{G}\left(A \cap G-\overline{\mathscr{C}}_{A}\right)=0
$$

and

$$
\mu_{U}\left(A \cap U-\overline{\mathscr{D}}_{A}\right)=0 .
$$

So, $\mathscr{C}_{A}^{*}=\mathscr{C}_{A} \cup \mathscr{D}_{A}^{\prime \prime}$ is a countable subfamily of $\mathscr{C}^{*}$ which verifies:

$$
\begin{aligned}
\mu_{G^{\prime}}\left(A-\overline{\mathscr{C}}_{A}^{*}\right) & \leq \mu_{G^{\prime}}\left(A \cap G-\overline{\mathscr{C}}_{A}^{*}\right)+\mu_{G^{\prime}}\left[A \cap(U-G)-\overline{\mathscr{C}}_{A}^{*}\right] \\
& \leq \mu_{G^{\prime}}\left(A \cap G-\overline{\mathscr{C}}_{A}\right)+\mu_{U}\left[A \cap(U-G)-\overline{\mathscr{D}}_{A}^{\prime \prime}\right] \\
& \leq \mu_{G^{\prime}}\left(A \cap G-\overline{\mathscr{C}}_{A}\right)+\dot{\mu}_{U}\left[A \cap(U-G)-\overline{\mathscr{D}}_{A}\right] \\
& \leq \mu_{G}\left(A \cap G-\overline{\mathscr{C}}_{A}\right)+\dot{\mu}_{U}\left(A \cap U-\overline{\mathscr{D}}_{A}\right) \\
& =0
\end{aligned}
$$

and, consequently, $\mathscr{C}^{*}$ verifies $\mathrm{M}_{2}$ and the lemma is proved because $\mathscr{C} \subset$ $\mathscr{C}^{*}$ by construction.

It should be notice that it follows from $\mathrm{M}_{2}$ that for every $H \in \mathscr{H}$ there exists a family $\mathscr{S}_{A} \subset \mathscr{D}_{A}$ such that

$$
\mu\left(A \cap U \cap H-\overline{\mathscr{S}}_{A}\right)=0
$$

and

$$
\begin{aligned}
\mu_{U}\left[A \cap(U-G) \cap H \cap \overline{\mathscr{S}}_{A}\right] & =\dot{\mu_{U}}\left[A \cap(U-G) \cap H \cap \overline{\mathscr{S}}_{A}^{\prime}\right] \\
& =\sum_{K \in \mathscr{S}_{A}^{\prime}} \mu_{U}[A \cap(U-G) \cap H \cap K] \\
& =\sum_{K \in \mathscr{Y}_{A}^{\prime \prime}} \mu_{U}[A \cap(U-G) \cap H \cap K] \\
& =\dot{\mu_{U}}\left[A \cap(U-G) \cap H \cap \overline{\mathscr{S}}_{A}^{\prime \prime}\right],
\end{aligned}
$$

therefore the inequality

$$
\dot{\mu_{U}}\left[\left(A \cap(U-G)-\overline{\mathscr{D}}_{A}^{\prime \prime}\right) \cap H\right] \leq \dot{\mu_{U}}\left[\left(A \cap(U-G)-\overline{\mathscr{D}}_{A}\right) \cap H\right]
$$

holds, and it follows from Theorem 74.2 of [7] that

$$
\dot{\mu_{U}}\left[A \cap(U-G)-\overline{\mathscr{D}}_{A}^{\prime \prime}\right] \leq \dot{\mu}_{U}\left[A \cap(U-G)-\overline{\mathscr{D}}_{A}\right] .
$$

Theorem 2. Every locally strictly localizable Radon measure of type $(\mathscr{H})$ on $E$, is strictly localizable.

Proof. Let $\mu$ be a locally strictly localizable Radon measure of type $(\mathscr{H})$ on $E$ and let us consider the set $\mathscr{A}$ of all pairs $(G, \mathscr{C})$ where $G$ is an open subset of $E$, such that $\mu_{G}$ is strictly localizable and $\mathscr{C}$ is a family 
of strict localizability for $\mu_{G}$. We consider in $\mathscr{A}$ the following order:

$$
\left(G_{1}, \mathscr{C}_{1}\right) \leq\left(G_{2}, \mathscr{C}_{2}\right) \Leftrightarrow G_{1} \subset G_{2} \text { and } \mathscr{C}_{1} \subset \mathscr{C}_{2} \text {. }
$$

We will see that if $\left\{\left(G_{i}, \mathscr{C}_{i}\right)\right\}_{i \in I}$ is a chain in $(\mathscr{A}, \leq)$ then $\mathscr{C}=\cup_{i \in I} \mathscr{C}_{i}$ is a family of strict localizability for $\mu_{G}$, being $G=\cup_{i \in I} G_{i}$, and therefore $(\mathscr{A}, \leq)$ is inductive.

If $A \subset G$ and $\mu_{G}(A)<+\infty$ then $A$ is $\mu_{G}$-compact and there is a countable subset $I^{\prime}$ of $I$ such that

$$
\mu_{G}\left(A-\bigcup_{i \in I^{\prime}} G_{i}\right)=0 .
$$

For every $i \in I^{\prime}$ we have that $\mu_{G_{i}}\left(A \cap G_{i}\right)<+\infty$ and there exists a countable subfamily $\mathscr{C}_{i}^{*}$ of $\mathscr{C}_{i}$ such that

$$
\mu_{G_{i}}\left(A \cap G_{i}-\overline{\mathscr{C}}_{i}^{*}\right)=0
$$

holds. Consequently $\mathscr{C}^{*}=\bigcup_{i \in I^{\prime}} \mathscr{C}_{i}^{*}$ is a countable subset of $\mathscr{C}$ such that

$$
\begin{aligned}
\mu_{G}\left(A-\overline{\mathscr{C}}^{*}\right) & =\mu_{G}\left[\left(A \cap \bigcup_{i \in I^{\prime}} G_{i}\right)-\overline{\mathscr{C}}^{*}\right] \\
& \leq \sum_{i \in I^{\prime}} \mu_{G_{i}}\left(A \cap G_{i}-\overline{\mathscr{C}}_{i}^{*}\right) \\
& =0
\end{aligned}
$$

and $\mathrm{M}_{2}$ holds. Therefore $\mathscr{C}$ is a family of strict localizability for $\mu_{G}$ and $(G, \mathscr{C}) \in \mathscr{A}$.

From Zorn's axiom it is deduced the existence of a maximal element $(G, \mathscr{C}) \in \mathscr{A}$ and it follows from Lemma 1 that $E-G$ is $\mu$-negligible.

Corollary 3. Every locally $\sigma$-finite Radon measure of type ( $\mathscr{H}$ ) on $E$ is strictly localizable.

Proof. It is an immediate consequence of Theorem 2, because every $\sigma$-finite measure is strictly localizable.

Proposition 4. Let $\mu$ be a Radon measure of type $(\mathscr{H})$ on $E$ and $\mathscr{C}$ a family of strict localizability for $\mu$, then we have:

4.1. If $A \subset E$ is such that $A \cap K$ is $\mu$-negligible for all $K \in \mathscr{C}$, then $A$ is $\mu$-negligible.

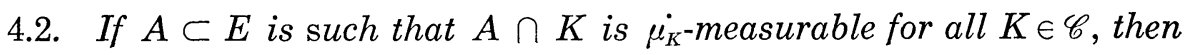
$A$ is $\mu$-measurable.

Proof. 4.1. For every $H \in \mathscr{H}$ there exists a countable subclass $\mathscr{C}_{H}$ of $\mathscr{C}$ such that $\mu\left(H-\overline{\mathscr{C}}_{H}\right)=0$ and 


$$
\begin{aligned}
\mu(A \cap \overline{\mathscr{C}} \cap H) & \leq \sum_{K \in \mathscr{C}_{H}} \mu(A \cap K \cap H) \\
& =0
\end{aligned}
$$

holds. Therefore it follows from Theorem 74.2 of [7] that

$$
\begin{aligned}
\mu(A \cap \overline{\mathscr{C}}) & =\sup \{\mu(A \cap \overline{\mathscr{C}} \cap H): H \in \mathscr{H}\} \\
& =0
\end{aligned}
$$

and $\mu(A)=0$.

4.2. For every $H \in \mathscr{H}$ there exists a countable subclass $\mathscr{C}_{H}$ of $\mathscr{C}$ such that $\mu\left(H-\overline{\mathscr{C}}_{H}\right)=0$. Consequently,

$$
\begin{aligned}
& \mu(H)=\mu\left(H \cap \overline{\mathscr{C}}_{H}\right) \\
& =\sum_{K \in \mathscr{Q}_{H}} \mu(H \cap K) \\
& =\sum_{K \in \mathscr{Q}_{H}}[\mu(H \cap K \cap A)+\mu((H-A) \cap K) \\
& =\mu(H \cap A)+\mu(H-A)
\end{aligned}
$$

and it follows from Theorem 75.2 of [7] that $A$ is $\mu$-measurable.

Remark 5. If $\mu$ is a Radon measure of type $(\mathscr{H})$ and $\mathscr{C}$ is a family of strict localizability for $\mu$, then there exists a family $\mathscr{C}^{\prime}$, of strict localizability for $\mu$, such that $\mathscr{C}^{\prime} \subset \mathscr{H}$ and every $K^{\prime} \in \mathscr{C}^{\prime}$ is contained in some $K \in \mathscr{C}$,

\section{REFERENCES}

[1] Chatterji, S. D., Desintegration of measures and liftings, Proc. of the Symposium on vector and operator valued measures and applications, Acad. Press, New York (1973), 69-83.

[ 2 ] Ionescu Tulcea, A. and C., Topics in the theory of lifting, Springer, Berlin, 1969.

[ 3 ] Maharam, D., On a theorem of von Neuman, Proc. Amer. Math. Soc., 9 (1958), 187-994.

[4] Neumann von, J., Algebraische Reprasentaten der Funktionen bis auf eine Menge von Masse Null, J. Crelle, 165 (1931), 109-115.

[5] Rodríguez-Salinas, B., Teoría de la medida sobre los espacios topológicos no localmente compactos, Rev. Mat. Hispano-Amer., (4), 32 (1973), 257-274.

[6] - $\mu$-espacios de Suslin y Lusin. Propiedad del lifting fuerte, Rev. R. Acad. Ci. Madrid, 72 (1978), 541-557.

[7] Rodríguez-Salinas, B. and P. Jiménez Guerra, Medidas de Radon de tipo ( $\mathscr{H}$ ) en espacios topológicos arbitrarios, Mem. R. Acad. Ci. Madrid, t. X, 1979.

[8] - Espacios de Radon de tipo ( $\mathscr{H}$ ), Rev. R. Acad. Ci. Madrid, 69 (1975), 761774.

[ 9 ] Ryan, R., Representative sets and direct sums, Proc. Amer. Math. Soc., 15 (1964), 387-390. 
[10] Schwartz, L., Radon measures on arbitrary topological spaces and cylindrical measures, Oxford University Press, 1973.

[11] Segal, I. E., Equivalences of measure spaces, Amer. J. Math., 73 (1951), 275-313.

Dpto. de Teoría de Funciones

Facultad de C. Matemáticas

Universidad Complutense

Madrid-3 (SPAIN) 